\title{
Recursos mineros en las Américas : mutaciones de un continente
}

Mineral resources in the Americas - a continent under mutation

Ressources minières dans les Amériques: Mutations d'un continent

Susanne Berthier-Foglar, Franck Gaudichaud y Sandrine Tolazzi

Traductor: Marta Gómez

\section{(2) OpenEdition}

\section{Journals}

Edición electrónica

URL: https://journals.openedition.org/ideas/3096

DOI: 10.4000/ideas.3096

ISSN: 1950-5701

Este artículo es una traducción de:

Ressources minières dans les Amériques: Mutations d'un continent - URL : https:// journals.openedition.org/ideas/1636 [fr]

Otras traducciones del artículo:

Mineral resources in the Americas - a continent under mutation - URL : https:// journals.openedition.org/ideas/2268 [en]

Recursos minerais nas Américas. Mutações de um continente - URL : https://

journals.openedition.org/ideas/4709 [pt]

Editor

Institut des Amériques

\section{Referencia electrónica}

Susanne Berthier-Foglar, Franck Gaudichaud y Sandrine Tolazzi, «Recursos mineros en las Américas : mutaciones de un continente», IdeAs [En línea], 8 | 2016, Publicado el 13 diciembre 2016, consultado el 18 octubre 2022. URL: http://journals.openedition.org/ideas/3096 ; DOI: https://doi.org/10.4000/ideas. 3096

Este documento fue generado automáticamente el 18 octubre 2022.

\section{cc) $(\mathrm{S})$}

Creative Commons - Atribución-NoComercial-SinDerivadas 4.0 Internacional - CC BY-NC-ND 4.0

https://creativecommons.org/licenses/by-nc-nd/4.0/ 


\title{
Recursos mineros en las Américas: mutaciones de un continente
}

\author{
Mineral resources in the Americas - a continent under mutation \\ Ressources minières dans les Amériques: Mutations d'un continent \\ Susanne Berthier-Foglar, Franck Gaudichaud y Sandrine Tolazzi \\ Tradución : Marta Gómez
}

1 En un contexto en el que la emergencia del cambio climático como amenaza mundial difícilmente puede frenar el desproporcionado consumo energético y la carrera por la energía que en gran medida constituyen la causa de dicho cambio, la cuestión del desarrollo de los recursos mineros y energéticos se perfila como un problema aún más acuciante si se tiene en cuenta que su producción ha aumentado significativamente tanto en el continente americano como en el resto del mundo. En los últimos años, el «boom » del extractivismo, entendido como actividad humana capaz de modificar de forma notable el medio ambiente y el clima, puede considerarse parte integrante de un conjunto de factores generadores de una nueva era geológica iniciada a principios del siglo XIX y bautizada con el nombre de " antropoceno " por el ganador del Nobel Paul Crutzen. Al atribuir un origen humano a los cambios del clima o del medio ambiente, los seguidores de la tesis del antropoceno, como Christophe Bonneuil y Jean-Baptiste Fressoz en su obra L'Événement anthropocène (París: Seuil, 2013), invitan a tomar conciencia del impacto de estas actividades en los cambios mundiales, sugiriendo que actualmente se ha alcanzado un punto sin retorno. Pese a que algunos miembros de la comunidad científica se niegan todavía a admitir el origen antropogénico de estos cambios (Samuele Furfari, en el artículo de Sandrine Tolazzi "Recursos mineros y energéticos: el choque de discursos»), la gran mayoría insiste en la necesidad de vincular, en términos generales, la cuestión de las consecuencias medioambientales con la de la explotación de recursos (Normand Mousseau, en el artículo citado). Por otra parte, si bien es cierto que la cuestión medioambiental centra a menudo los debates actuales sobre el desarrollo de los recursos mineros, también parece que el impacto económico, político, geoestratégico y social del auge reciente de las industrias extractivas en el continente americano está generando una auténtica mutación en 
dicho continente, tal y como ilustra la entrevista a Bernadette Mérenne-Schoumaker. Por ello y ante todo para determinar la naturaleza de estas mutaciones, los coordinadores de este número de IdeAs organizaron en un primer momento un coloquio internacional sobre la cuestión de los recursos mineros en las Américas (Grenoble, junio de 2014) para posteriormente solicitar la colaboración de algunos participantes en dicho coloquio y de otros autores con el fin de elaborar un dossier que, esperan, sea representativo de los cuestionamientos científicos actuales. La lectura de las distintas contribuciones, artículos de vigilancia científica y entrevistas en torno a estas cuestiones hace patentes numerosas zonas de tensión ligadas a juegos de poder entre diferentes actores (comunidades locales, empresas, gobiernos a diferentes escalas, etc.) pero también a las representaciones de estos juegos de poder relacionadas a veces con la historia del desarrollo minero en general.

2 La problemática de la explotación minera -y más ampliamente, de los recursos naturales- ocupa un lugar destacado dentro de los grandes cambios geopolíticos y la redefinición asimétrica de las relaciones Norte-Sur: la apropiación, extracción y control de las riquezas que genera esta actividad han sido importantes motores de los procesos coloniales y de expansión territorial que han vivido las Américas desde los siglos XV y XVI. La "sed» de oro y plata ha alimentado una larga experiencia de dominación socioeconómica, política y cultural, más allá incluso de las Independencias, forjando hasta nuestros días una suerte de «tradición » de explotación de recursos de índole colonial, a través de la cual las economías latinoamericanas extrovertidas se han vuelto dependientes de las grandes potencias industriales que dominan el sistema internacional (véase « Domination et extraversion de l'Amérique latine » en Géopolitique des Amériques, dir. Alain Musset, París : Nathan, 2014, 4 ed.). Dicho modelo se plasma de norte a sur del continente, además de reflejarse en las relaciones de América Latina con otros polos de la economía mundial (Europa, China), e incluso a nivel interno dentro de los países, tanto al norte como al sur del continente. Así, la autora Lucie Genay («Extracción de uranio y perpetuación del colonialismo interno en Nuevo México ») muestra hasta qué punto la historia de la extracción de uranio en este Estado confirma que, si bien los retos de la actividad minera han cambiado, siguen prevaleciendo algunos esquemas neocoloniales de la construcción nacional estadounidense, perpetuando una herencia de desigualdad social y racial y generando a su vez reacciones desiguales por parte de las naciones indias implicadas. Este «neocolonialismo extractivista " también es denunciado con firmeza por numerosos movimientos sociales y medioambientales y comunidades indígenas de América Latina, unas movilizaciones que han dado origen a diversos conflictos con los gobiernos en el poder y las empresas mineras. Frente a la proliferación de dichas movilizaciones, los gobiernos del continente han ensayado diversas estrategias, desde la represión o la contención institucional hasta el establecimiento de moratorias o la declaración de partes del territorio nacional como libres de explotación minera (María Teresa Gutiérrez Haces, « La inversión extranjera y la actividad minera en América Latina. Las estrategias de los gobiernos en los conflictos con las compañías mineras »). En efecto, ya se definan como progresistas, "nacional-populares » o conservadores, todos los gobiernos han intentado sacar provecho del «boom » del precio de las materias primas y mineras de los últimos años, aunque sea según lógicas a menudo distintas en términos de políticas públicas. No obstante, la crisis mundial y la caída de la demanda desde 2007-2008 han puesto de manifiesto hasta qué punto las economías americanas podían enmarcarse en una lógica de renta y reprimarización contraria a cualquier perspectiva 
de desarrollo "sostenible » o virtuoso. Así pues, ¿es posible otro modelo minero ? Así parece sugerirlo el gobierno bolivariano de Venezuela con su «Plan de acción estratégica común para el desarrollo del cinturón y el arco minero del Orinoco » con el que pretende articular crecimiento económico, alianza con las empresas multinacionales y desarrollo de servicios sociales públicos, «controlando » al mismo tiempo la evolución del pionero frente minero. Con todo, el estudio de Anne PénéAnnette sobre la extracción aurífera en el estado Bolívar ("¿Reactivación de la extracción minera en la Guayana venezolana?») subraya la magnitud de la "destrucción medioambiental y los daños humanos" que puede suponer este extractivismo llevado a cabo a escala de todo un país.

3 En la actualidad son muchos los investigadores y activistas que no solo proponen análisis críticos de estas formas de explotación industrial de la naturaleza sino que además hacen hincapié en modelos alternativos de relación con la naturaleza, la sociedad y la economía, e incluso las relaciones Norte-Sur (véase el artículo de Sandrine Tolazzi, "Recursos mineros y energéticos: el choque de discursos ", el artículo de Maristella Svampa y la obra de Anna Bednik citados en Franck Gaudichaud, « Recursos mineros, extractivismo y desarrollo en América Latina : perspectivas críticas »). Así, en Ecuador, el concepto del buen vivir (o Sumak Kawsay, en quechua), o la idea de dejar especialmente en zonas de gran biodiversidad- los minerales o el petróleo bajo tierra empiezan a ganar terreno en la sociedad civil y entre los responsables políticos, sin que ello resuelva sin embargo la problemática tensión entre la protección de los ecosistemas, el respeto a las comunidades y el acceso a un cierto nivel de vida para todas y todos. Así, en la Bolivia de Evo Morales, si bien el imaginario del buen vivir constituye un referente simbólico al que recurre con frecuencia el Presidente, el país sigue dependiendo fuertemente de la explotación de los recursos mineros y de gas cuyo desarrollo actual obedece más a las presiones de diferentes organizaciones corporativas que a la reconquista de la soberanía nacional anunciada (Claude Le Gouill, « La política minera del gobierno de Evo Morales : entre mitos y pragmatismo político »).

4 La cuestión medioambiental también es un componente esencial de la historia del desarrollo minero que, en opinión de Michel Deshaies, ha tenido siempre un carácter « depredador » para el medio ambiente (« Minas y medio ambiente en las Américas : las paradojas de la explotación minera»). Ciertamente, los pequeños prospectores y empresas mineras que explotaban los recursos sin restricciones con miras a obtener una rentabilidad inmediata y que luego dejaban abandonados unos terrenos altamente contaminados han desaparecido en beneficio de grandes sociedades mineras cuya actividad está actualmente más controlada. Por otra parte, las presiones de algunas comunidades locales y ONG han llevado a estas empresas a reducir su impacto medioambiental y, a su vez, a implicarse más en el desarrollo de los territorios en cuestión con el objetivo de aumentar su aceptabilidad e intentar revertir la imagen negativa que podían proyectar. En esta línea, Deshaies destaca que paradójicamente, las grandes empresas mineras se topan con una oposición cada vez mayor debido a su impacto medioambiental aun cuando éste nunca ha sido tan bajo. Con todo, no es menos cierto que la escala misma de los proyectos mineros que se desarrollan actualmente contribuye a que ese impacto siga siendo importante, sin mencionar la necesaria toma en consideración de los problemas relacionados con la rehabilitación de los territorios una vez finalizada su explotación, además del riesgo de catástrofes 
naturales, como recordó recientemente y de forma trágica la catástrofe minera de Mariana en el estado brasileño de Minas Gerais.

5 Se ha avivado por lo tanto el debate en torno a las consecuencias medioambientales de los megaproyectos mineros, obligando a los gobiernos a tomar mayores precauciones y realizar estudios tales como la evaluación medioambiental estratégica sobre gases de esquisto encargada por el Gobierno de Quebec y en la que participaron Christiane Gagnon y Majella-J. Gauthier ( Inventario y vulnerabilidad territorial de las tierras bajas de San Lorenzo (Quebec, Canadá) frente a la explotación de gas de esquisto »). Así, el trabajo presentado en este estudio ha permitido detectar diversas zonas de vulnerabilidad, lo que debería favorecer una mejor gestión del territorio en el marco de proyectos de explotación en Quebec. Sin embargo, para los científicos sigue siendo difícil posicionarse al margen de cualquier discurso sobre tales cuestiones. Por un lado, se perfila claramente una retórica que trata de minimizar el impacto de los grandes proyectos de desarrollo, incluso de presentarlos como beneficiosos para el medio ambiente. En este sentido, Samuele Furfari no solo afirma que « la energía fósil, a pesar de su impacto medioambiental, ha tenido repercusiones extraordinariamente positivas en nuestras condiciones de trabajo, vida y salud» sino que además presenta el desarrollo del gas de esquisto como una solución para reducir los gases de efecto invernadero (Sandrine Tolazzi, "Recursos mineros y energéticos: el choque de discursos»). En consecuencia, a los detractores del desarrollo de este gas se les presenta por ejemplo como personas mal informadas sobre los procesos de exploración y extracción (RTI International, en el artículo citado). En el otro extremo se distingue un discurso que denuncia claramente el impacto medioambiental del desarrollo de los recursos mineros, presentándolo como uno de los principales desafíos a la hora de elaborar una estrategia mundial sobre dichos recursos (Normand Mousseau, en el artículo citado). La cuestión que debe dilucidarse es si cabe centrarse en reducir el impacto medioambiental de los proyectos mineros o bien en suprimir pura y simplemente dichos proyectos en el marco de un proceso de transición ecológica.

6 En otro orden de cosas, la explotación de recursos moviliza a un número cada vez mayor de actores locales que han tomado conciencia de su condición de minoría explotada, e incluso sacrificada. La ubicación de yacimientos en zonas de montaña que históricamente han servido de refugio a poblaciones pobres, en su mayor parte indígenas, ha llevado a estos habitantes a tomar conciencia de la expoliación de la que han sido objeto desde hace siglos. Las etnias locales han adquirido pues una nueva visibilidad dentro de la escena política al oponerse a los dirigentes de un Estado que perciben como parte de un grupo social y cultural distinto. En América Latina se observa un resurgir de antiguas identidades étnicas silenciadas en un contexto de asimilación y denigración de lo "indio ", término que en español puede tener un sentido peyorativo. Desde hace unos quince años, las comunidades campesinas, a menudo indígenas en el sentido dado por la Declaración de las Naciones Unidas sobre los derechos de los pueblos indígenas, han ejercido una oposición creciente a algunos proyectos mineros y han exigido a las empresas mineras mayores beneficios a nivel local, así como el derecho a participar de los mismos. El artículo de Carmen Salazar Soler « La importancia de la etnicidad en los conflictos mineros socioambientales en los Andes peruanos durante los siglos XX y XXI » pone de relieve esta toma de conciencia así como las demandas de la población indígena en materia de control eficaz de la 
contaminación y de participación en las decisiones sobre la explotación y la utilización de los recursos naturales y sus territorios.

7 Para Lucie Genay («Extracción de uranio y perpetuación del colonialismo interno en Nuevo México»), la industria extractiva en tierras de indígenas y poblaciones hispánicas de Nuevo México se asemeja a la vez a una conquista y a una colonización. Genay pone de manifiesto la vulnerabilidad de los habitantes de estas regiones mineras cuya economía se ve debilitada por el carácter cíclico de la explotación de estos recursos. Desde un punto de vista social, la autora también destaca la persistencia de una herencia de dependencia y desigualdades pese a la fuerte militancia que, aunque sin cohesión centralizadora, se está organizando en las regiones donde se ubican los yacimientos de uranio.

8 La militancia ecológica está muy arraigada desde hace años en el oeste de los Estados Unidos donde de hecho se gestó, coincidiendo con los movimientos de conservación de la naturaleza y la creación de los primeros parques nacionales a finales del siglo XIX. Esta tradicional visión de la naturaleza está presente en el activismo de oposición a las explotaciones mineras. Un lugar con una fuerte visibilidad desde el punto de vista medioambiental, ya sea un parque nacional o cualquier otra zona protegida, incluso una montaña sagrada, cristaliza el rechazo a la explotación minera. Todo esto es especialmente evidente si se analizan las contundentes opiniones de las tribus-naciones Navajo y Laguna (Susanne Berthier-Foglar, «La explotación de los recursos mineros en el oeste de Estados Unidos: el uranio, los indígenas y los ambientalistas », y Lucie Genay, "Extracción de uranio y perpetuación del colonialismo interno en Nuevo México »).

9 Más allá del contexto estadounidense, diversas organizaciones militantes de ámbito internacional se interesan por las situaciones de expoliación de pueblos oprimidos en el mundo. Lejos del contexto social y económico, estas tomas de posición no siempre surten gran efecto pero sirven al menos para alertar a la opinión pública. En cualquier caso, en los países estudiados, es evidente que las razones económicas o militares priman sobre la protección de la naturaleza. El artículo de Susanne Berthier-Foglar («Recursos mineros y energéticos : evolución y desafíos constantes») relata cómo los propietarios de una mina de uranio, en pleno corazón del parque nacional del Gran Cañón, obtuvieron luz verde para la explotación intrusiva del lugar, situado a poca distancia de un importante emplazamiento turístico. Durante la Guerra Fría, las cuestiones de defensa nacional y la carrera por las armas nucleares parecían más importantes que la integridad de un parque.

10 Así pues, la cuestión de la explotación de los recursos mineros y energéticos es fundamental a la hora de comprender ciertos modelos económicos de desarrollo, pero también algunas relaciones de poder en juego. Dicha cuestión es tanto más fascinante para los investigadores que se interesan por ella cuanto que plantea problemáticas a todos los niveles, desde el individuo -como puede observarse a través de las fotografías de Louie Palu (entrevistada por Lucie Genay)- a la comunidad, pasando por actores gubernamentales e industriales, con un impacto económico y medioambiental que puede evaluarse a nivel local pero también regional e incluso mundial. Se trata por tanto de una cuestión verdaderamente estratégica en el contexto de las Américas. Los artículos seleccionados aquí constituyen una primera presentación de algunos aspectos ligados a este amplio campo de investigación. 


\section{AUTORES}

\section{SUSANNE BERTHIER-FOGLAR}

Susanne Berthier-Foglar est professeur, spécialiste de l'Ouest américain et des peuples autochtones, Université Grenoble Alpes (ILCEA4). susanne.berthier@univ-grenoble-alpes.fr

\section{FRANCK GAUDICHAUD}

Franck Gaudichaud est maître de conférences en histoire et civilisation de l'Amérique Latine à l'Université Grenoble Alpes et membre de l'ILCEA4 (EA 7356). franck.gaudichaud@univ-grenoblealpes.fr

\section{SANDRINE TOLAZZI}

Sandrine Tolazzi est maître de conférences à l'Université Grenoble Alpes (UFR des Langues, ILCEA4 - Institut des Langues et des Cultures d'Europe, Amérique, Afrique, Asie et Australie). sandrine.tolazzi@univ-grenoble-alpes.fr 